\title{
RELATO DE EXPERIÊNCIA: DESAFIOS NA PROMOÇÃO DE SAÚDE ÀS GESTANTES ADOLESCENTES ACOMPANHADAS NA ATENÇÃO SECUNDÁRIA
}

\author{
EXPERIENCE REPORT: CHALLENGES IN HEALTH PROMOTION TO \\ ADOLESCENT PREGNANT WOMEN ACCOMPANIED IN SECONDARY CARE
}

\begin{abstract}
Ana Cindy de Souza Fonteles *, Camila Albuquerque de Queiroza, Camilla Araújo Lopes Vieiraa, Leidiana do Nascimento Pintoa, Luiz Odorico Monteiro de Andradea, Roberlandia Evangelista Lopesa ${ }^{\text {, Lidyane Parente Arruda }}{ }^{a}$
\end{abstract}

Universidade Federal do Cearáa E-mail: ana_cindy1@hotmail.com

\section{RESUMO}

Promoção de Saúde (PS) é um conjunto de práticas de saúde que buscam evitar a exposição às doenças numa perspectiva ampliada de saúde. As adolescentes gestantes de alto risco apresentam diversas vulnerabilidades, necessitam de acolhimento e encaminhamentos para se sentirem seguras nos serviços de assistência, considerando que maternidade na adolescência traz alterações biopsicossociais. Esse estudo tem o objetivo de analisar os desafios da PS a esse público por uma enfermeira assistente no protocolo de alto risco de gestantes de uma unidade da atenção secundária no município de Sobral - Ceará. Trata-se de um relato de experiência em um serviço ambulatorial de obstetrícia da atenção secundária, foram analisadas adolescentes acompanhadas no período de janeiro a junho de 2020. Os desafios presentes no cuidado as jovens gestantes levantam questões enfrentadas pela equipe multiprofissional, apontando necessidade de constantes formações aos profissionais de saúde e fortalecimento da rede de cuidado a esse público, fatores relevantes identificados a partir desse relato.

Palavras-chave: Promoção de Saúde; Gravidez; Gravidez de Alto Risco.

\section{ABSTRACT}

Health Promotion is a set of health practices that seek to avoid exposure to diseases in an expanded health perspective. High-risk pregnant adolescents have several vulnerabilities, need reception and referrals to feel safe in care services, considering that adolescent motherhood brings biopsychosocial changes. This study aims to analyze the challenges of health promotion to this audience by an assistant nurse in the right-risk protocol for pregnant women in a secondary care unit in the municipality of Sobral - Ceará. This is an experience report in an outpatient midwifery outpatient service, adolescents monitored in the period from January to June 2020 were analyzed training for health professionals and strengthening the care network for this public, relevant factor identified from this report.

Keyword: Health Promotion; Pregnancy; High-Risk Pregnancy. 


\section{INTRODUÇÃO}

Promoção da Saúde (PS) pode ser definida como um conjunto de práticas, programas e estratégias políticas de saúde com o objetivo de evitar que pessoas se exponham a fatores, condicionantes e determinantes considerados adoecedores no âmbito individual ou coletivo, pensando de maneira ampla, para além da prevenção de doenças, deve-se também considerar a dimensão histórica e social de cada indivíduo. Apontando as práticas de saúde que ocorrem nas redes de atenção como um vínculo revigorante para adolescentes gestantes, busca-se fazer uma reflexão sobre a PS em sua vertente crítica que tem como foco modificar positivamente as condições de vida e saúde dos processos individuais e/ou coletivos (RIBEIRO; SANTOS; FRANCALINO; MENDES, 2019).

Juventude é um tema que vem ganhando espaço e representatividade no meio acadêmico e social, seja pelo grande número populacional ou pelas necessidades sociais em torno desse público. Cerca de $27 \%$ da população brasileira se encontram na faixa etária dos 15 aos 29 anos, compreendida pelo Estado brasileiro como uma população economicamente ativa que necessita de políticas públicas especificas (BARREIRO; MALFITANO, 2017).

Dados da Prefeitura Municipal de Sobral apontam que a cidade tem cerca de $33,3 \%$ da população composta por jovens de 15 a 29 anos, compreendendo um total de 66 mil jovens. Por isso, há a necessidade de que sejam garantidos vários direitos durante essa etapa, deste modo ver-se necessária uma compressão da juventude como uma fase de experimentações e de formação individual e coletiva, na perspectiva de ultrapassar as visões tradicionais, que enxergam as juventudes com uma mera fase de transição entre infância e a idade adulta ou uma fase problemática.

É fundamental a ampliação de políticas afirmativas de oferta de serviços e atividades, bem como, ampliação do protagonismo juvenil e emancipação dos e das jovens, na busca de uma perspectiva transversal para as políticas públicas municipais como saúde, educação e cultura.

A discussão sobre juventudes e saúde fornece subsídios para a compreensão de Políticas Públicas para as Juventudes, no setor Saúde, entendendo a saúde como não somente ausência de doença, mas, principalmente, como grande cenário para construção de saberes, no sentido de estruturar ações assertivas dentro do campo do cuidado à saúde. Muitas ações ocuparam a agenda pública nos últimos anos. O principal marco é o Estatuto da Juventude, sancionado pela Presidente Dilma em 2013, que tem como objetivo instituir os direitos dos jovens e das jovens, por meio dos princípios e das diretrizes para as ações políticas nacionais a serem garantidos ao jovem brasileiro (BARREIRO; MALFITANO, 2017), principalmente às mulheres, considerando suas especificidades e diversidade. $\mathrm{O}$ referido estatuto, discorre que o jovem tem direito à saúde e à qualidade de vida, considerando suas especificidades na dimensão da prevenção, promoção, proteção e recuperação da saúde de forma integral. Ao mesmo tempo, a assistência às mulheres em período gravídico é uma prerrogativa do SUS nos vários níveis de assistência (MARTINS et al, 2020).

A gravidez na adolescência pode ser apontada como um desafio para o setor saúde, considerando alterações que podem repercutir na vida da jovem gestante, precocemente inserida no mundo adulto ao exercício da maternidade, podendo acarretar graves consequências que afetam a própria adolescente, bebê e todos ao redor, relacionadas aos aspectos biológicos e sociais tais como prematuridade, aumento da morbidade e mortalidade infantil, aumento de situações de abandono, violências, além de evasão escolar, dificuldade de ingresso no mercado de trabalho, rejeição familiar e o desenvolvimento de transtornos mentais, como depressão e ideação suicida (CUNHA et al, 2020).

Esses desafios trazem a reflexão sobre a importância de se discutir sobre a maternidade, principalmente quando acontece na adolescência é um assunto que deve ser debatido na sociedade, encarando as mudanças que ocorrem na rotina da mulher, do bebê, da família e a comunidade em que aquelas pessoas se inserem, é preciso compreender como as mulheres compreendem esse papel de mãe, como se manifesta esse papel na vida delas, se já era algo almejado.

A ideia de que maternidade pode ser uma escolha não é um fato muito aceito pela sociedade. A mulher é cotidianamente cobrada pela sociedade a exercer o papel de mãe, sendo estimulada por estruturas sociais tradicionais como a família, o estado, o mercado do capital e a igreja, que acabam por supor a capacidade produtiva e reprodutiva de cada mulher como fator intrínseco, biológico, natural e inevitável. É comum a circulação de discursos que trazem a vida de uma mulher associada simbolicamente ao exercício da maternidade, mesmo que elas próprias hesitem sobre o desejo em cumprir esse papel (GONZAGA; MAYORGA, 2019). Esse discurso repercute na atenção em saúde e no manejo 
do cuidado às adolescentes em período de gestação, tendo em vista a formação incipiente sobre o tema nos ambientes de formação dos profissionais. Tal aspecto impacta diretamente na forma de acolhimento e no acompanhamento e cuidado, podendo refletir e transparecer desconforto no profissional de saúde e na mulher atendida nos espaços. Podemos ter aí um distanciamento clínico entre profissional de saúde e usuários, que repercute diretamente no manejo do cuidado e nos índices de adesão ao pré-natal.

É possível analisar que os temas oficiais das ações desenvolvidas pelos dos profissionais de saúde com as jovens estão quase sempre relacionados a gravidez na adolescência e também algumas Infecções Sexualmente Transmissíveis (IST), no entanto, muitas vezes, sem uma reflexão mais ampla que discutam questões que evidenciam os temas de gênero e sexualidade.

Os discursos e naturalização de que a mulher representa uma beleza, fragilidade e maternal acaba por reforçar a privação das jovens em espaços importantes para seu desenvolvimento pessoal e social como trabalho, estudos e vida social, inclusive em participação comunitária. Nesse contexto é possível identificar uma grande lacuna no que diz respeito às manifestações femininas juvenis, e no âmbito da saúde, restringem-nas apenas à vinculação ao exercício da sexualidade ou a gravidez. Há questionamentos sobre a fragilidade das políticas públicas criadas para o incentivo da participação social do sexo feminino, levando em consideração ações especificas que respondam suas demandas e necessidades.

Para discutir questões acerca da promoção de saúde, maternidade e adolescência pode se analisar a superação de limitações atribuídas para a PS, que apresentam a forma de como eles são postos em prática nos serviços da Atenção Primária, além de trazer uma reflexão sobre a PS em sua vertente crítica que tem como foco a transformação das condições de vida e saúde de indivíduos e coletivos (CARVALHO; COHEN; AKERMAN, 2017). Os autores nos guiam para uma avaliação do antagonismo da prevenção e da PS, ou seja, que a PS seria mais ampla e complexa que a prevenção. Critica a prevenção de doenças que têm uma visão biologista e comportamentalista do processo-saúdedoença, não considerando a dimensão histórica e social do processo.

De forma geral, na literatura, identificam-se duas tendências dentre os discursos de promoção da saúde. A primeira coloca em destaque o estilo de vida e é focada no indivíduo ou, no máximo, na sua família. A segunda, considerada a moderna promoção da saúde pautada na Carta de Ottawa, leva em conta a qualidade de vida baseada nos determinantes gerais das condições de vida e saúde da sociedade e é voltada ao coletivo e ao ambiente (Verdi \& Caponi, 2005; Haeser, Buchele, \& Brzozowski, 2012; Penido \& Romagnoli, 2018).

Evidencia-se que essa questão, é cada vez mais presente na atualidade, precisa ser discutida, em diversos setores, e requer uma demanda de olhares, principalmente da abordagem de gênero do setor saúde, considerando que a saúde é vinculada à cidadania, autonomia na construção na forma de viver, logo comprometida com equidade e justiça social (CARVALHO; COHEN; AKERMAN, 2017).

Assim apostar em tecnologias leves é buscar um modelo amparado na integralidade, é preciso transitar pelo território subjetivo do trabalho vivo em ato na saúde, potencializando redes vivas do cuidado e modos de produzir acolhimento, sem deixar de considerar a importância das demais tecnologias (SANTOS; MISHIMA; MERHY, 2018). Para os autores as tecnologias leves se apresentam como prática de acolhimento, escuta e diálogo, significando campo privilegiado para o exercício do autogoverno e de reaproximação do fazer em saúde com os projetos de vida das pessoas.

Entendendo que a saúde da mulher jovem tem representado um desafio para os profissionais de saúde que acolhem este grupo, principalmente em espaços de assistência que acolhem gestantes de alto risco, esse relato tem como objetivo analisar os desafios da PS a esse público por uma enfermeira assistente no protocolo de alto risco de gestantes de uma unidade da atenção secundária no município de Sobral - Ceará, buscando apontar estratégias de cuidado em uma atuação interdisciplinar e multiprofissional por meio de programas de atenção integral pautados na política de PS na atenção secundária.

O Sistema de Saúde de Sobral é composto por uma rede de atenção à saúde hierarquizada e regionalizada com serviços em diferentes níveis de complexidade, sendo o Polo para a Macrorregião Norte do Ceará. Esta rede compreende 249 estabelecimentos e serviços relacionados à Atenção Primária, Atenção Especializada e Atenção Terciária à Saúde, conforme o cadastro Nacional de Estabelecimentos de Saúde (CNES, 2017), distribuídos na rede pública e privada do município. Fonte: Plano Municipal de Saúde - 20172020.

Sendo a atenção secundária formada pelos serviços especializados em nível ambulatorial e 
hospitalar, com densidade tecnológica intermediária entre atenção primária e a terciária, historicamente interpretada como executora de procedimentos de média complexidade que compreende serviços médicos especializados, de apoio diagnóstico e terapêutico, além de atendimentos em urgência e emergência (MEDEIROS et al, 2019). Os serviços prestados por essas unidades atendem os principais problemas da população abrangida e demandam profissionais especializados, além do uso de tecnologia como apoio para o diagnóstico e terapêutico operados por médicos especialistas e outros profissionais da saúde, planejado para agilizar o acesso e aumentar a resolutividade de consultas e procedimentos especializados (BRASIL, MINISTÉRIO DA SAÚDE, 2019).

\section{METODOLOGIA}

\section{Aspectos éticos e tipo de estudo}

O estudo seguiu os aspectos éticos, respeitando os princípios da bioética preconizados pela Resolução 466/2012. Trata-se de uma pesquisa descritiva exploratória, do tipo relato de experiência. O relato de experiência tem como característica a observação sistemática da realidade a partir do acompanhamento de gestantes por uma profissional enfermeira, no protocolo de alto risco.

\section{Desenho, local e período do estudo}

O estudo foi desenvolvido por meio da observação, em um serviço ambulatorial de obstetrícia da atenção secundária, referência para usuárias do Sistema Único de Saúde - SUS, localizado no município de Sobral. Nesse ambulatório é desenvolvido o Protocolo de Gestante do Alto Risco vinculado a um hospital de referência da cidade. O acompanhamento é feito por equipe multidisciplinar, composto por profissionais obstetra, enfermeira, nutricionista e psicóloga e consiste em consultas individuais e atividades educativas em sala de espera, espaço que se destina. A pesquisa foi realizada no período de janeiro a junho de 2020.

\section{Participantes do estudo}

Gestantes adolescentes que foram encaminhadas pela APS, sendo reguladas pelo sistema de regulação Fast Medic - Excelência na Gestão em Saúde do Estado do Ceará. Consultou-se os registros dos prontuários eletrônicos das gestantes para identificar os critérios de elegibilidade. Os critérios de inclusão foram gestantes com a idade mínima de 15 anos e máxima de 18 anos de idade incompletos. Como critérios de exclusão, gestantes que receberem alta do serviço ambulatorial.

\section{RESULTADOS E DISCUSSÃO}

É possível afirmar que a partir da vivência e observações em um serviço ambulatorial de obstetrícia da atenção secundária, que o acompanhamento de gestantes de alto risco é um desafio para todos os níveis de complexidade. É factível observar a existência de vários fatores que influenciam nessa realidade: a necessidade de acompanhamento desde o início da gestação pela Atenção Primária e o devido encaminhamento para os níveis de média e alta complexidade, assim como a identificação e ativação de uma rede de cuidados para essas com acompanhamento de diversos setores, grupos de apoio, orientação e aproximação da família nesse processo, identificando assim que agravos biopsicossociais podem ser evitados e cuidados em tempo hábil (FERREIRA et al,2019).

Os desafios existentes no cuidado as jovens gestantes identificadas como alto risco levantam várias questões que são difíceis de serem abordadas pelas equipes de cuidado. A dificuldade em temas considerados delicados e dificuldades no estabelecimento de vínculo podem ser um empecilho no acompanhamento e assistência a essas mulheres. Conforme o gráfico pode-se notar a não adesão das mesmas a continuidade do pré-natal na atenção secundária. 
Gráfico 1. Quantidade de gestantes vinculadas ao Protocolo de Alto Risco (janeiro a junho de 2020), em porcentagem representa o quantitativo das adolescentes. Os dados são retirados dos mapas de atendimentos dos meses.

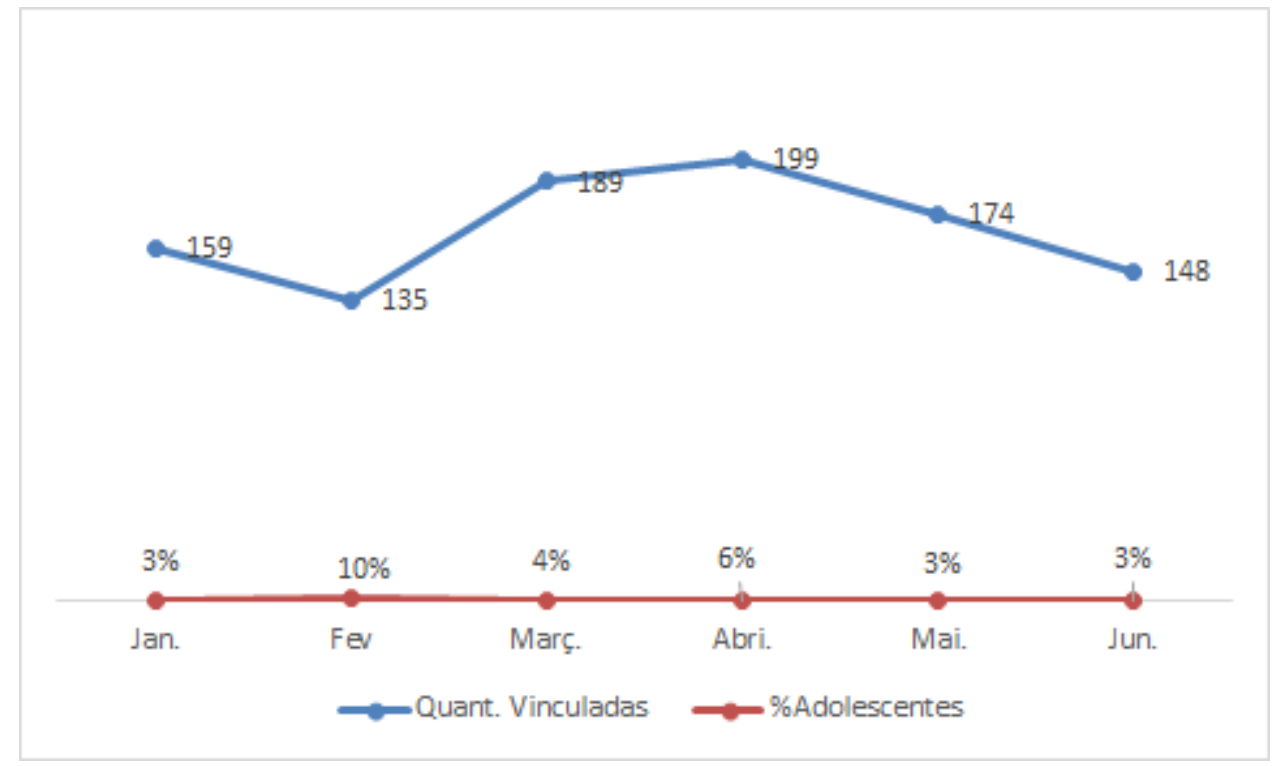

Fonte: Sistema Integrado de Gestão em Saúde, 2020.

Os encaminhamentos realizados para pré-natal de alto risco na unidade secundária ocorrem por meio da APS, a partir do momento em que o médico da unidade básica considera a possibilidade de complicações relativas a doenças preexistentes ou intercorrências da gravidez gerados por fatores orgânicos, socioeconômicos e demográficos desfavoráveis. A avaliação obstétrica ocorre em cada consulta individualmente, sendo o retorno agendado conforme a necessidade clínica, além de exames complementares como cardiotocografia, ultrassom obstétrica com/ou sem doppler, transvaginal, entre outros, registrados no Sistema Integrado em Gestão de Saúde - SIGES e no prontuário físico.

As ações de promoção da saúde na unidade são realizadas semanalmente, conforme os agendamentos. Os momentos acontecem nas salas de espera da unidade, com temas variados, que vão desde a apresentação do protocolo ao esclarecimento do agendamento realizados pelas gestantes/parceiros/familiares para conhecerem a atenção terciária.

Essas ações de PS são encaradas como momentos onde as gestantes podem expressar seus sentimentos, a partir do investimento de diálogo pelos profissionais de saúde tornado esses momentos de assistência mais humanizados, percebendo que momentos de trocas envolvendo os acompanhantes e colocando as gestantes como proativas, tornam essas jovens mais próximas dos profissionais de saúde, conseguindo um investimento maior em seus planos de cuidados, tornando uma atenção mais horizontal e efetiva no cuidado dessas adolescentes (FERREIRA et al,2019).

A partir da vivência no serviço ambulatorial foi perceptível a identificação de que as gestantes que estabeleciam uma relação de confiança já na atenção primária, a partir dos atendimentos conseguiam seguir os planos de cuidados pactuados de maneira mais comprometida, apontando a importância dos momentos de PS em toda rede de atenção a esse público, revelando que as adolescentes acompanhadas que estabelecem uma relação de vínculo com os profissionais do Centro de Saúde da Família, conseguem se sentir mais seguras nos serviços de maior complexidade, fator que se deve há uma aproximação maior entre profissionais de saúde e a jovem gestante.

O bem-estar espiritual é considerado uma dimensão do estado de saúde das pessoas e foi defendido por Wanda de Aguiar Horta, ao propor a Teoria das Necessidades Humanas Básicas. Dessa forma, tal necessidade também deve ser priorizada nas consultas do pré-natal de alto risco e podem influenciar, positivamente, na vinculação a equipe/unidade ou criar obstáculos na adesão aos mesmos. Quando analisamos esse estabelecimento de vínculo e empatia na rede de atenção a essas jovens, se aponta que a Política Nacional 
de Humanização veio justamente propor mudanças no processo de trabalho e nas relações entre profissionais de saúde e as pessoas que buscam cuidado nos diversos serviços de saúde, objetivando que a proposta de um atendimento humanizado objetive principalmente o bem estar físico, psíquico e social dos usuários dos serviços de saúde, necessitando que os profissionais envolvidos no cuidado ofereçam uma maior qualidade nos atendimentos deixando os usuários satisfeitos com a assistência em saúde (PANTOJA; BRITO; SILVA, 2020).

Profissionais da área da saúde envolvidos no cuidado às gestantes tem um papel fundamental em garantir que essas mulheres tenham uma assistência pautada nos aspectos da humanização na assistência que se trata de manter um relacionamento baseado em confiança, respeito e empatia durante os atendimentos de saúde, em especial as gestantes adolescentes que ao viver a experiência da gestação podem enfrentar diversos conflitos, necessitando assim que seus direitos sejam exercidos de forma humanizada na assistência em saúde (PANTOJA; BRITO; SILVA, 2020).

A criação dos momentos relacionais às gestantes e seus acompanhantes favorece trocas de experiências e facilita a abordagem dos assuntos pontuados na sala de espera. Contudo, diante das observações, foi constatado que o pré-natal tem pouca aderência, com reflexo na não presença nas consultas e resistência à nova rotina $\mathrm{e}$ realidade que a gravidez impõe no cuidado em saúde materno-infantil.

É preciso identificar que esses contextos influenciam em como a rede de atenção às gestantes de alto risco precisa ser cada vez mais fortalecida com programas, políticas de formação permanente dos profissionais de referência e a necessidade do fortalecimento do apoio matricial na Atenção Primária que identifiquem as vulnerabilidades e melhore a comunicação intersetorial entre os serviços e níveis de complexidade. A partir da vivência relatada é possível dizer que as vulnerabilidades territoriais vivenciadas pelos sujeitos da pesquisa necessitam de uma articulação da rede, bem como da utilização de protocolos melhor definidos pelos fluxos vivenciados no município (FERNANDES et al, 2019).

A integração entre serviços, instituições e organizações, grupos e sujeitos com a finalidade de acompanhar uma demanda de saúde, por trocas de experiências e efetivação de políticas públicas pode ser definida como Redes de Atenção em Saúde, com estrutura à integralidade na atenção em saúde de forma qualificada (EVANGELISTA et al, 2020).

$\mathrm{O}$ acompanhamento integral e o acionamento das redes de atenção às gestantes adolescentes só são possíveis com a sensibilização dos profissionais, com a formação constante em Educação Permanente (EP) tendo em vista que sua função é promover aprendizagem significativa aos profissionais de saúde de maneira contínua, com práticas de reflexão e problematização com o objetivo de lidar e mudar as realidades vivenciadas (CAMPOS; SENA; SILVA, 2017). Pontua-se a necessidade da existência dessa prática de problematização como meio de mudança da realidade na rede de atenção à adolescentes gestantes de alto risco.

\section{CONCLUSÃO}

Com base em aspectos observados em um serviço ambulatorial de assistência à gestantes adolescentes consideradas de alto risco no período de janeiro a julho de 2020 é possível afirmar que diversos desafios fazem parte desse cenário, considerado significativo problema para a saúde pública, em especial na atenção primária, por ser considerada a mantedora do elo paciente e rede de atenção.

O nível de atenção especializada geralmente exige mais recursos do que a atenção primária porque é ressaltado o uso e desenvolvimento de tecnologias/exames específicos para conclusão do diagnóstico e proporcionar o melhor tratamento para o paciente. Desenvolver e manter um protocolo de alto risco na atenção secundária foi elaborado devido as caraterísticas peculiares que a rede secundaria proporciona.

A gravidez não transforma apenas o corpo/físico da gestante, mas também repercuti e podemos até confirmar que noventa por cento o emocional da mulher. Imaginemos juntar essas explosões de emoções ao período da fase da adolescência. Repercussões significativas na vivência gestacional, impactos sócio emocionais perante a sociedade e a própria família, não sem efeito à família nuclear aos que estão no entorno relacional, no aspecto social das pessoas envolvidas nesse cenário, na vida futura da mulher, além do acréscimo da pandemia em relação a Covid-19, que comprovadamente foi apresentado aos brasileiros que o Sistema Único de Saúde não estava amadurecido para enfrentar uma pandemia. Portanto, o sistema de saúde está diante de um cenário desafiador tanto para o manejo do cuidado quanto para o investimento em tecnologia relacional.

A partir de tais aspectos é necessário discutir sobre tornar a assistência à essas jovens mulheres, uma assistência de melhor qualidade e mais humanizada, valorizando o respeito no cuidado prestado. A gravidez, 
especificamente na adolescência modifica a rotina dessas jovens e de seus familiares, os momentos de PS na atenção secundária colaboram para proporcionar, além do cuidado, o apoio e o envolvimento dos acompanhantes no processo de cuidar. É por meio da transformação nesse cuidado que surge uma atenção mais horizontalizada, efetiva, ética e responsável.

O presente relato de experiência também faz refletir que é necessário o fortalecimento das redes de apoio a essas jovens gestantes em todos os níveis de complexidade e na constante Educação Permanente pautada na Política Nacional de Promoção à Saúde, com investimento na constante formação dos profissionais de saúde que estão inseridos na rede de cuidado, independentemente do nível de complexidade do serviço de assistência prestado. E que o reconhecimento de uma necessidade é um passo relevante para a busca da minimização do problema, pois, se os questionamentos/necessidades não forem visualizados/reconhecidos, não haverá progresso positivo, além de que o papel fundamental do profissional de saúde é promover a saúde da população, apoiar os que buscam e até os que por algumas dificuldades/obstáculos desistiu da assistência, que por direito devem receber.

\section{AGRADECIMENTOS}

A Universidade pela oportunidade de fazer o curso de Pós-Graduação, ao seu corpo docente, direção e administração que oportunizaram a janela que hoje vislumbramos um horizonte superior, pautado pela confiança no mérito e ética aqui presentes.

\section{REFERÊNCIAS}

BARREIRO, R.G \& MALFITANO, A. P. S. (2017). Política brasileira para a juventude: a proposta dos Centros da Juventude. Revista Latinoamericana de Ciências Sociales, Niñez y Juventud, 15 (2), pp. 111111122. DOI:10.11600/169271x.1522113122016.

BRASIL, Ministério da Saúde. Caderno de Parâmetros para Programação das Ações e Serviços de Saúde de Média e Alta Complexidade, no âmbito do Sistema Único de Saúde - SUS. 2019. Disponível em: < http://www.saude.campinas.sp.gov.br/programas/protoc olos/Parametros_SUS_2015.pdf> Acesso em: 30 out. 2020.

CAMPOS, K. F. C; SENA, R. R; SILVA, K. L. Educação Permanente nos serviços de saúde. EEAN, Minas Gerais, v. 4, n. 21, p.1-10, abr. 2017.

CUNHA, Alyne Condurú dos Santos et al. Efeitos psicossociais da gravidez na adolescência: um estudo transversal. Brazilian Journal of Development, Curitiba, v. 6, n. 7, p. 47412-47424, Jul. 2020. Disponível em:

https://www.brazilianjournals.com/index.php/BRJD/arti cle/view/13283/11161. Acesso em: 27 set. 2020.

EVANGELISTA, Maria José de Oliveira et al. O Planejamento e a construção das Redes de Atenção à Saúde no DF, Brasil. Ciência \& Saúde Coletiva [online], Brasília, v. 24, n. 6, p. 2115-2124. Disponível em: <https://doi.org/10.1590/1413-

81232018246.08882019>. ISSN 1678-4561.Acesso em 27 de setembro. 2020.

FERNANDES, Juliana Azevedo et al. Avaliação da atenção à gestação de alto risco em quatro metrópoles brasileiras. Cadernos de Saúde Pública [online]. v. 36, n. 5, e00120519. ISSN 1678-4464. Disponível em: <https://doi.org/10.1590/0102-311X00120519>. Acesso em: 26 set. 2020. Doi: https://doi.org/10.1590/0102$\underline{311 \times 00120519}$

FERREIRA, S. V. et al. Cuidado de enfermagem na ótica das gestantes de alto risco. REFACS, Uberaba, MG, v. $7, \quad$ n. 2, p. 143-150, 2019. DOI: 10.18554/refacs.v7i2.3410. Disponível em:< http://seer.uftm.edu.br/revistaeletronica/index.php/refac s>. Acesso em: 27 set. 2020.

GONZAGA, Paula Rita Bacellar; MAYORGA, Claudia. Violências e Instituição Maternidade: uma Reflexão Feminista Decolonial. Psicol. Cienc. prof., Brasília, v. 39, n. spe2, e225712, $2019 . \quad$ Disponível em: <http://www.scielo.br/scielo.php?script=sci_arttext\&pid $=$ S1414-98932019000600307\&lng $=$ en\&nrm $=$ iso $>$.

Acesso em: 12 set. de 2020. 
LAGO, P. N.; SOUSA, A. A. S; RODRIGUES, D. P SILVA, M. R. F; MESQUITA, N. S. A atenção primária em saúde como fonte de apoio social a gestantes adolescentes. Enferm Bras 2019;18(1):75-84. Disponível em: $<$ http://dx.doi.org/10.33233/eb.v18i1.2480>. Acesso em: 12 set. 2020.

MARTINS, Ana Beatriz Albuquerque Almeida; VIEIRA, Camilla Araújo Lopes. Corpo e subjetividade na vivência gestacional: um estudo com mulheres em Sobral-CE. Ecos | Estudos Contemporâneos da Subjetividade, [S.I.], v. 10, n. 1, p. 23-33, jan. 2020. Disponível em: http://www.periodicoshumanas.uff.br/ecos/issue/view/2 67/showToc. Acesso em: 08 nov. 2020.

MEDEIROS, Fabiana Fontana et al. Acompanhamento pré-natal da gestação de alto risco no serviço público. Rev. Bras. Enferm. Brasília, v. 72, supl. 3, p. 204-211, 2019.

Disponível em:<http://www.scielo.br/scielo.php?script=sci_arttext \&pid=S0034-71672019000900204\&lng=en\&nrm=iso >. Acesso em: 08 nov. 2020. Disponível em: <https://doi.org/10.1590/0034-7167-2018-0425>.

Acesso em: 15 set. 2020.

PANTOJA, Eurides Duque; BRITO, Leidiane Santos de; SILVA, Adan Renê Pereirada. A GESTÃO DO ATENDIMENTO HUMANIZADO À GESTANTE EM MANAUS -AMAZONAS. Rech-Revista Ensino de Ciências e Humanidades -Cidadania, Diversidade e Bem Estar, Manaus, v. 6, n. 1, p. 177-191, jun. 2020. Disponível em: <https://periodicos.ufam.edu.br/index.php/rech/article/vi ew/7568/5312>. Acesso em: 25 set. 2020.

RIBEIRO, Geiciara Costa; SANTOS, Francisco Edinardo de Carvalho; FRANCALINO, Terezinha Ribeiro; MENDES, Igor Cordeiro. UTILIZAÇÃO DE TECNOLOGIAS DE PROMOÇÃO DA SAÚDE PELOS ENFERMEIROS NA ATENÇÃO PRIMÁRIA. Encontro de Extensão, Docência e Iniciação Científica (Eedic), [S.L.], v. 5, n. 1, p. 1-8, mar. 2019. Disponível em:

http://publicacoesacademicas.unicatolicaquixada.edu.br/ index.php/eedic/article/view/3148/2697. Acesso em: 30 out.

2020s://periodicos.ufam.edu.br/index.php/rech/article/vi ew/7568. Acesso em: 30 out. 2020.
PENIDO, C. M. F. \& ROMAGNOLI, R. C. Apontamentos sobre a clínica da autonomia na promoção da saúde. Psicologia \& Sociedade, 30, e173615, 2018. Disponível em: <http://dx.doi.org/10.1590/18070310/2018v30173615>. Acesso em: 30 out. 2020.

SANTOS, Debora de Souza; MISHIMA, Silvana Martins; MERHY, Emerson Elias. Processo de trabalho na Estratégia de Saúde da Família: potencialidades da subjetividade do cuidado para reconfiguração do modelo de atenção. Ciênc. Saúde coletiva, Rio de Janeiro, v. 23, n. 3, p. 861-870, mar. 2018 . Disponível em: $<$ http://www.scielo.br/scielo.php?script=sci_arttext\&pid $=$ S1413-81232018000300861\&lng=en\&nrm=iso $>$.

Acesso em: 08 nov. 2020. Doi: https://doi.org/10.1590/1413-81232018233.03102016.

SILVA, Anitha de Cássia Ribeiro da et al. IMPORTANCIA DO PRE NATAL NA OPINIÃO DAS USUÁRIAS DE UMA UNIDADE BASICA DE SAÚDE DA FAMILIA EM PORTO VELHO, RONDÔNIA. Revista Saber Científico, Porto Velho, v. 8, n. 2, p. 89 - 98, dez. 2019. ISSN 1982-792X. Disponível em: <http://revista.saolucas.edu.br/index.php/resc/article/vie w/1240>. Acesso em: 27 out. 2020. doi: http://dx.doi.org/10.22614/resc-v8-n2-1240.

SOBRAL, Prefeitura de. Plano Municipal de Saúde de Sobral. Sobral, 2017-2020. Acesso em: <https://divulgacandcontas.tse.jus.br/dados/2016/CE/13 447/2/60000015302/proposta_governo1471299181659. pdf>. Acesso em: 20 set. 2020. 